\title{
Adaptive Output Feedback Compensation of Variant Actuator Failures *
}

\author{
Gang Tao \\ Department of Electrical and Computer Engineering \\ University of Virginia \\ Charlottesville, VA 22903
}

\author{
Suresh M. Joshi \\ Mail Stop 132 \\ NASA Langley Research Center \\ Hampton, VA 23681
}

\begin{abstract}
This paper presents an adaptive output feedback control scheme for systems with uncertain actuator failures whose values are variant. An effective controller structure is developed for adaptive actuator failure compensation using a new parametrization scheme. A new failure compensation property is derived for such a scheme which leads to a linear parametrization of the bilinear parameters caused by plant and failure uncertainties. Desired plant-model matching, stable adaptation and asymptotic output tracking properties are established for the closed-loop control system in the presence of variant actuator failures. Simulation results verified the desired adaptive actuator failure compensation performance.
\end{abstract}

Keywords: Actuator failure, adaptive compensation, parametrization, stability and tracking, variant failure model.

\section{Introduction}

System component failures often cause severe feedback control system performance deterioration. Effective failure compensation is critical for many applications such as flight control systems. Different failure compensation approaches have been developed including multiple models, switching and tuning designs [4], adaptive designs [1], [2], [3], [8], [11], fault diagnosis, detection and tolerant designs [5], [7], [9], [10], [12]. Such specifically designed control systems have not only robust stability but also tracking performance guarantees, despite the presence of system failures.

For actuator failure compensation, a desirable controller should be able to adjust its parameters to accommodate actuator failure uncertainties including failure time, patterns and values. A basic and reasonable assumption is that there exists a nominal controller to meet a control objective if the actuator failures were known. Adaptation can then be employed to handle the case when the failure are unknown. The key feature of an adaptive design is that it ensures asymptotic tracking in the presence of uncertain actuator failures. Recently, we developed direct adaptive state feedback control schemes for linear time-invariant plants with actuator failures

\footnotetext{
*This research was partially supported by NASA Langley Research Center under grant NCC-1-02006.
}

characterized by inputs stuck at some values not influenced by control action.In [8], we derived and studied an adaptive output feedback compensation scheme for actuator failures whose values are constant and unknown. The goal of this paper is to develop an adaptive output feedback compensation scheme for actuator failures whose values are variant and unknown. The control problem formulation is given in Section 2. To handle such actuator failures, a new controller parametrization is needed to linearly parametrize bilinear parameters of the dynamic feedforward part of the model reference controller as well as the uncertain actuator failures. Such a new parametrization is first illustrated in Section 3 for adaptive output rejection of uncertain variant input disturbances, and then developed in Section 4 for adaptive compensation of uncertain variant actuator failures using output feedback. The adaptive law for updating the controller parameters is designed to ensure closed-loop system stability and asymptotic output tracking. The desired performance of our adaptive actuator failure compensation scheme is verified in Section 5 by simulation results from adaptive yaw rate control of a linearized Boeing 747 lateral dynamic model with actuator failures in a multi-segment rudder servomechanism.

\section{Problem Formulation}

Consider the actuator failure compensation problem for

$$
\dot{x}(t)=A x(t)+B u(t), y(t)=c x(t),
$$

where $A \in R^{n \times n}, B=\left[b_{1}, \ldots, b_{m}\right] \in R^{n \times m}$, and $c \in R^{1 \times n}$ are unknown constant parameter matrices, $u(t)=\left[u_{1}, \ldots, u_{m}\right]^{T} \in$ $R^{m}$ is the input vector whose components may fail during system operation, and $y(t) \in R$ is the plant output.

The variant actuator failure model is

$$
\begin{array}{r}
u_{j}(t)=\bar{u}_{j}(t)=\bar{u}_{j 0}+\sum_{l=1}^{q} \bar{d}_{j l} f_{j l}(t), t \geq t_{j}, \\
j \in\left\{j_{1}, j_{2}, \ldots, j_{p}\right\} \subset\{1,2, \ldots, m\},
\end{array}
$$

where $\bar{u}_{j 0}$ and $\bar{d}_{j l}$, are unknown constant parameters, and $f_{j l}(t)$ are known bounded signals, $l=1,2, \ldots, q$.

The input signals $u_{i}(t)$ are described by

$$
u_{i}(t)=v_{i}(t)+\sigma_{i}(t)\left(\bar{u}_{i}(t)-v_{i}(t)\right), i=1,2, \ldots, m,
$$


where $\sigma_{i}(t)=1$ if the $i$ th actuator has failed, i.e., $u_{i}(t)=\bar{u}_{i}(t)$, since $t_{i} \leq t$ and $\sigma_{i}(t)=0$ otherwise, and $v_{i}(t)$ are the applied control input signals. The key feature of the control problem is that the failure pattern $\sigma_{i}(t), i=1,2, \ldots, m$, is unknown.

The control objective is to find the control signals $v_{i}(t)$, $i=1,2, \ldots, m$, using output feedback, such that the plant output $y(t)$ asymptotically tracks a reference output $y_{m}(t)$ generated from the reference model system

$$
y_{m}(t)=W_{m}(s)[r](t), W_{m}(s)=\frac{1}{P_{m}(s)},
$$

in addition to closed-loop stability, where $P_{m}(s)$ is a stable monic polynomial of degree $n^{*}$, and $r(t)$ is bounded.

In many applications, the redundant actuators $u_{i}(t), i=$ $1,2, \ldots, m$, are desired to generate proportional effects on the system dynamic behavior (for example, in aircraft flight control, different rudder segments have proportional effects on aircraft lateral motion). A proportional actuation scheme

$$
v_{i}(t)=\alpha_{i} v_{0}(t), \alpha_{i}>0, i=2,3, \ldots, m,
$$

is an appropriate choice, with $v_{0}(t)$ to be designed.

A nominal controller structure for $v_{0}(t)$ is

$$
\begin{aligned}
& v_{0}(t)=v_{0}^{*}(t) \\
= & \theta_{1}^{* T} \omega_{1}(t)+\theta_{2}^{* T} \omega_{2}(t)+\theta_{20}^{*} y(t)+\theta_{3}^{*} r(t)+\theta_{4}^{*}(t),
\end{aligned}
$$

where $\theta_{1}^{*} \in R^{n-1}, \theta_{2}^{*} \in R^{n-1}, \theta_{20}^{*} \in R$, and $\theta_{3}^{*} \in R$ are parameters for plant-model output matching, $\theta_{4}^{*}(t)$ is a time-varying signal for compensating the variant failures, and

$$
\omega_{1}(t)=\frac{a(s)}{\Lambda(s)}\left[v_{0}^{*}\right](t), \omega_{2}(t)=\frac{a(s)}{\Lambda(s)}[y](t)
$$

with $a(s)=\left[1, s, \ldots, s^{n-2}\right]^{T}$ and $\Lambda(s)$ being a monic stable polynomial of degree $n-1$. Based on the chosen controller structure, $\theta_{1}^{*}, \theta_{2}^{*}, \theta_{20}^{*}, \theta_{3}^{*}$ and $\theta_{4}^{*}$ can be specified, dependent on the knowledge of the plant and actuator failure parameters, to meet the control objective. The existence of such $\theta_{1}^{*}, \theta_{2}^{*}$, $\theta_{20}^{*}, \theta_{3}^{*}$ and $\theta_{4}^{*}$ is important for an adaptive design for the case when the plant and failure parameters are unknown.

Suppose that at a certain time instant $t$, there are $p$ failed actuators, that is, $u_{j}(t)=\bar{u}_{j}, j=j_{1}, \ldots, j_{p}, 1 \leq p<m$. From (2.3) and (2.5), we can express the plant (2.1) as

$$
y(t)=G(s)\left[v_{0}\right](t)+\bar{y}(t)
$$

where

$$
G(s)=\frac{\sum_{j \neq j_{1}, \ldots, j_{p}} \alpha_{j} k_{p j} Z_{j}(s)}{P(s)} \triangleq \frac{k_{p} Z_{a}(s)}{P(s)}
$$

for $C(s I-A)^{-1} b_{j}=k_{p j} Z_{j}(s) / P(s), j=1,2, \ldots, m$, with $k_{p j}$ a scalar parameter, $Z_{j}(s)$ a monic polynomial, and $P(s)$ a monic polynomial of degree $n$, and for some equivalent scalar parameter $k_{p}$ and monic polynomial $Z_{a}(s)$, and

$$
\bar{y}(t)=\sum_{j=j_{1}, \ldots, j_{p}} \frac{\alpha_{j} k_{p j} Z_{j}(s)}{P(s)}\left[\bar{u}_{j}\right](t) .
$$

For plant-model output matching and closed-loop system stability, we make the following assumptions:

(A.1) $Z_{a}(s)$ has degree $n-n^{*}$, i.e., the systems ( $C, A$, $\left.\sum_{j \neq j_{1}, \ldots, j_{p}} \alpha_{j} b_{j}\right), p \in\{0,1, \ldots, m-1\}$, have the same relative degree $n^{*}$ and are minimum phase (i.e., $Z_{a}(s)$ is stable).

Determining the parameters $\theta_{1}^{*}, \theta_{2}^{*}, \theta_{20}^{*}, \theta_{3}^{*}$ from

$$
\begin{aligned}
& \theta_{1}^{* T} a(s) P(s)+\left(\theta_{2}^{* T} a(s)+\theta_{20}^{*} \Lambda(s)\right) k_{p} Z_{a}(s) \\
= & \Lambda(s)\left(P(s)-k_{p} \theta_{3}^{*} Z_{a}(s) P_{m}(s)\right)
\end{aligned}
$$

with $\theta_{3}^{*}=k_{p}^{-1}$, we can express the closed-loop system as

$$
\begin{aligned}
& \theta_{3}^{*} P_{m}(s)\left[y-y_{m}\right](t)= \\
& \theta_{4}^{*}(t)+\left(1-\theta_{1}^{* T} \frac{a(s)}{\Lambda(s)}\right) \frac{\sum_{j=j_{1}, \ldots, j_{p}} \alpha_{j} k_{p j} Z_{j}(s)}{k_{p} Z_{a}(s)}\left[\bar{u}_{j}\right](t) .
\end{aligned}
$$

Since $\Lambda(s)$ and $Z_{a}(s)$ are stable, there exists a parametrized signal $\theta_{4}^{*}(t)$ (see Section 4.1) such that the second term in the right side of (2.12) converges to zero exponentially as $t \rightarrow \infty$, so that $\lim _{t \rightarrow \infty}\left(y(t)-y_{m}(t)\right)=0$ exponentially.

Let $\left(T_{i}, T_{i+1}\right), i=0,1, \ldots, m_{0}$, with $T_{0}=0$, be the time intervals on which the actuator failure pattern is fixed, that is, actuators fail only at time $T_{i}, i=1, \ldots, m_{0}$. Since there are $m$ actuators and at least one of them does not fail, we have $m_{0}<m$ and $T_{m_{0}+1}=\infty$. Then, at time $T_{j}, j=1, \ldots, m_{0}$, the unknown plant-model matching parameters $\theta_{1}^{*}, \theta_{2}^{*}, \theta_{20}^{*}, \theta_{3}^{*}$, and $\theta_{4}^{*}$, change their values so that they are actually piecewise constant parameters, because the plant has different characterizations under different failure conditions, leading to different plant-model matching parameters.

\section{Adaptive Disturbance Rejection}

To introduce the basic idea of adaptive compensation of variant actuator failures, we start with the following adaptive output feedback disturbance rejection problem for the plant

$$
\dot{x}(t)=A x(t)+b_{i} u_{i}(t)+b_{j} u_{j}(t), y(t)=c x(t)
$$

where $u_{i}(t)$ and $u_{j}(t) \in R$ are two actuating inputs. The problem is to design one (control) input $u_{i}(t)$ to cancel the effect of the other (disturbance) input $u_{j}(t)$ on the output $y(t)$.

Consider the input-output form of this plant, that is,

$$
y(t)=\frac{k_{p i} Z_{i}(s)}{P(s)}\left[u_{i}\right](t)+\frac{k_{p j} Z_{j}(s)}{P(s)}\left[u_{j}\right](t)
$$

where $u_{i}(t)$ is the control input and $u_{j}(t)$ is a disturbance of the form (2.2). The control objective is to find an output feedback control signal $u_{i}(t)$ such that $y(t)$ tracks $y_{m}(t)$, under the assumptions that $Z_{i}(s)$ is stable, $P(s)$ has degree $n$, and $k_{p i} Z_{i}(s) / P(s)$ has relative degree $n^{*}$, despite the presence of the uncertain, variant and parametrizable disturbance $u_{j}(t)$. 


\subsection{Nominal Control Parametrization}

For the nominal case when the plant parameters in $k_{p i} Z_{i}(s) / P(s)$ are known, we design the controller (2.6) with $u_{i}(t)=v_{0}(t)=v_{0}^{*}(t)$. The matching equation (2.11) becomes

$$
\begin{aligned}
& \theta_{1}^{* T} a(s) P(s)+\left(\theta_{2}^{* T} a(s)+\theta_{20}^{*} \Lambda(s)\right) k_{p i} Z_{i}(s) \\
= & \Lambda(s)\left(P(s)-k_{p i} \theta_{3}^{*} Z_{i}(s) P_{m}(s)\right)
\end{aligned}
$$

with $\theta_{3}^{*}=k_{p i}^{-1}$, from which we can derive

$$
\begin{aligned}
& \theta_{3}^{*} P_{m}(s)\left[y-y_{m}\right](t) \\
= & \theta_{4}^{*}(t)+\left(1-\theta_{1}^{* T} \frac{a(s)}{\Lambda(s)}\right) \frac{k_{p j} Z_{j}(s)}{k_{p i} Z_{i}(s)}\left[u_{j}\right](t) .
\end{aligned}
$$

From (3.3), there is a monic polynomial $P_{i}(s)$ of degree $n^{*}-1$, satisfying $\frac{\Lambda(s) P_{m}(s)}{P(s)}=P_{i}(s)+\frac{R(s)}{P(s)}$ for some polynomial $R(s)$ of degree $n-1$ such that

$$
\Lambda(s)-\theta_{1}^{* T} a(s)=Z_{i}(s) P_{i}(s)
$$

(and $\theta_{1}^{* T} a(s)+\theta_{20}^{*} \Lambda(s)=-\theta_{3}^{*} R(s)$ ), from which we express

$$
\left(1-\theta_{1}^{* T} \frac{a(s)}{\Lambda(s)}\right) \frac{k_{p j} Z_{j}(s)}{k_{p i} Z_{i}(s)}\left[u_{j}\right](t)=\frac{k_{i j} Z_{j}(s) P_{i}(s)}{\Lambda(s)}\left[u_{j}\right](t),
$$

where $k_{i j}=\frac{k_{p j}}{k_{p i}}$. Then, the choice of the compensation signal

$$
\theta_{4}^{*}(t)=-\frac{k_{i j} Z_{j}(s) P_{i}(s)}{\Lambda(s)}\left[u_{j}\right](t)
$$

leads (3.4) to the desired tracking error equation $\theta_{3}^{*} P_{m}(s)[y-$ $\left.y_{m}\right](t)=0$ (with closed-loop stability), under the condition that the degree of $Z_{j}(s)$ is not greater than that of $Z_{i}(s)$.

\subsection{Adaptive Controller Structure}

For the case when the plant parameters and actuator failure parameters are unknown, we use the adaptive controller

$$
u_{i}(t)=\theta_{1}^{T} \omega_{1}(t)+\theta_{2}^{T} \omega_{2}(t)+\theta_{20} y(t)+\theta_{3} r(t)+\theta_{4}(t),
$$

where $\theta_{1}, \theta_{2}, \theta_{20}, \theta_{3}$, and $\theta_{4}(t)$ are the estimates of $\theta_{1}^{*}, \theta_{2}^{*}$, $\theta_{20}^{*}, \theta_{3}^{*}$, and $\theta_{4}^{*}(t)$, respectively.

For the disturbance $u_{j}(t)$ in (2.2), we express

$$
u_{j}(t)=\phi_{j}^{* T} \psi_{j}(t)
$$

where

$$
\begin{aligned}
\phi_{j}^{*} & =\left[\bar{u}_{j}, \bar{d}_{j 1}, \ldots, \bar{d}_{j q}\right]^{T} \\
\psi_{j}(t) & =\left[1, f_{j 1}(t), \ldots, f_{j q}(t)\right]^{T} \in R^{q+1} .
\end{aligned}
$$

For $\theta_{4}^{*}(t)$ in (3.7), we express

$$
\begin{aligned}
\theta_{4}^{*}(t) & =-\frac{k_{i j} Z_{j}(s) P_{i}(s)}{\Lambda(s)}\left[u_{j}\right](t) \\
& =\theta_{i j}^{* T} \frac{a(s)}{\Lambda(s)}\left[u_{j}\right](t)+\theta_{i j 0}^{*} u_{j}(t)
\end{aligned}
$$

for some parameters $\theta_{i j}^{*} \in R^{n-1}$ and $\theta_{i j 0}^{*} \in R$ such that

$$
\theta_{i j}^{* T} \frac{a(s)}{\Lambda(s)}+\theta_{i j 0}^{*}=-\frac{k_{i j} Z_{j}(s) P_{i}(s)}{\Lambda(s)} .
$$

We then introduce the new parametrization

$$
\theta_{4}^{*}(t)=\theta_{5}^{* T} \omega_{5}(t)+\theta_{50}^{* T} \Psi_{j}(t)
$$

where

$$
\begin{aligned}
\theta_{5}^{*} & =\theta_{i j}^{*} \otimes \phi_{j}^{*}, \theta_{50}^{*}=\theta_{i j 0}^{*} \phi_{j}^{*} \\
\omega_{5}(t) & =\frac{A(s)}{\Lambda(s)}\left[\psi_{j}\right](t) \\
A(s) & =\left[I_{q+1}, s I_{q+1}, \ldots, s^{n-2} I_{q+1}\right]^{T}
\end{aligned}
$$

with $\theta_{i j}^{*} \otimes \phi_{j}^{*}=\left[\theta_{i j 1}^{*} \phi_{j}^{* T}, \ldots, \theta_{i j n-1}^{*} \phi_{j}^{* T}\right]^{T} \in R^{(n-1)(q+1)}$ being the Kronecker product of $\theta_{i j}^{*}=\left[\theta_{i j 1}^{*}, \ldots, \theta_{i j n-1}^{*}\right]^{T} \in R^{n-1}$ and $\phi_{j}^{*} \in R^{q+1}$. For the adaptive controller (3.8), we use

$$
\theta_{4}(t)=\theta_{5}^{T}(t) \omega_{5}(t)+\theta_{50}^{T}(t) \psi_{j}(t)
$$

where $\theta_{5}(t)$ and $\theta_{50}(t)$ are the estimates of $\theta_{5}^{*}$ and $\theta_{50}^{*}$.

Using (3.3), we can parametrize the plant (3.1) as

$$
\begin{aligned}
& \theta_{1}^{* T} \frac{a(s)}{\Lambda(s)}\left[u_{i}\right](t)+\theta_{2}^{* T} \frac{a(s)}{\Lambda(s)}[y](t)+\theta_{20}^{*} y(t)=u_{i}(t) \\
& -\theta_{3}^{*} P_{m}(s)[y](t)+\left(1-\theta_{1}^{* T} \frac{a(s)}{\Lambda(s)}\right) \frac{k_{p j} Z_{j}(s)}{k_{p i} Z_{i}(s)}\left[u_{j}\right](t) .
\end{aligned}
$$

Using (3.8) and (3.16), we obtain the error equation

$$
\theta_{3}^{*} P_{m}(s)\left[y-y_{m}\right](t)=\tilde{\theta}^{T}(t) \omega(t)
$$

where $\tilde{\theta}(t)=\theta(t)-\theta^{*}$,

$$
\begin{aligned}
\theta(t) & =\left[\theta_{1}^{T}(t), \theta_{2}^{T}(t), \theta_{20}(t), \theta_{3}(t), \theta_{5}^{T}(t), \theta_{50}^{T}(t)\right]^{T} \\
\theta^{*} & =\left[\theta_{1}^{* T}, \theta_{2}^{* T}, \theta_{20}^{*}, \theta_{3}^{*}, \theta_{5}^{* T}, \theta_{50}^{* T}\right]^{T} \\
\omega(t) & =\left[\omega_{1}^{T}(t), \omega_{2}^{T}(t), y(t), r(t), \omega_{5}^{T}(t), \psi_{j}^{T}(t)\right]^{T} .
\end{aligned}
$$

Based on the error equation (3.17), an estimation error can be defined for the development of an adaptive law for updating the parameter estimate $\theta(t)$ so that the desired closed-loop stability and asymptotic output tracking are ensured.

\section{Adaptive Failure Compensation}

Now an adaptive compensation control scheme is developed for the plant (2.1) with unknown plant parameters and unknown variant actuator failures (2.2).

\subsection{Nominal Control Parametrization}

To parametrize the nominal controller (2.6), we consider the system (2.12). Similar to (3.5), there exists a monic polynomial $P_{a}(s)$ of degree $n^{*}-1$ such that

$$
\Lambda(s)-\theta_{1}^{* T} a(s)=Z_{a}(s) P_{a}(s) .
$$


Then, we can choose the nominal compensation signal

$$
\theta_{4}^{*}(t)=-\sum_{j=j_{1}, \ldots, j_{p}} \frac{\alpha_{j} k_{p j} Z_{j}(s) P_{a}(s)}{k_{p} \Lambda(s)}\left[\bar{u}_{j}\right](t)
$$

to ensure the desired error equation $\theta_{3}^{*} P_{m}(s)\left[y-y_{m}\right](t)=0$, under Assumption (A.1) that $Z_{a}(s)$ has the same degree $n-n^{*}$ as $Z_{j}(s), j=j_{1}, j_{2}, \ldots, j_{p}$, which is needed (and necessary) for solving the failure compensation problem.

For the failure signals $\bar{u}_{j}(t), j=j_{1}, j_{2}, \ldots, j_{p}$, in (4.2), with $\phi_{j}^{*}$ and $\psi_{j}(t)$ defined in (3.9), we parametrize $\theta_{4}^{*}(t)$ as

$$
\begin{aligned}
\theta_{4}^{*}(t) & =-\sum_{j=j_{1}, \ldots, j_{p}} \frac{k_{p j} Z_{j}(s) P_{a}(s)}{k_{p} \Lambda(s)}\left[\bar{u}_{j}\right](t) \\
& =\sum_{j=1}^{m} \theta_{p j}^{* T} \frac{a(s)}{\Lambda(s)}\left[\bar{u}_{j}\right](t)+\sum_{j=1}^{m} \theta_{p j 0}^{*} \bar{u}_{j}(t)
\end{aligned}
$$

for some parameters $\theta_{p j}^{*} \in R^{n-1}$ and $\theta_{p j 0}^{*} \in R$, where $\theta_{p j}^{*}=0$, $\theta_{p j 0}^{*}=0$, for $j \neq j_{1}, j_{2}, \ldots, j_{p}$. Using the Kronecker product $\otimes($ see $(3.14))$, we define

$$
\theta_{6 j}^{*}=\theta_{p j}^{*} \otimes \phi_{j}^{*}, j=1,2, \ldots, m
$$

and introduce the new parametrization

$$
\theta_{4}^{*}(t)=\theta_{6}^{* T} \omega_{6}(t)+\theta_{60}^{* T} \omega_{60}(t)
$$

where

$$
\begin{aligned}
\theta_{6}^{*} & =\left[\theta_{61}^{* T}, \ldots, \theta_{6 m}^{* T}\right]^{T} \in R^{m(n-1)(q+1)} \\
\theta_{60}^{*} & =\left[\theta_{p 10}^{*} \phi_{1}^{* T}, \ldots, \theta_{p m 0}^{*} \phi_{m}^{* T}\right]^{T} \in R^{m(q+1)} \\
\omega_{6}(t) & =\left[\left(\frac{A(s)}{\Lambda(s)}\left[\psi_{1}\right](t)\right)^{T}, \ldots,\left(\frac{A(s)}{\Lambda(s)}\left[\psi_{m}\right](t)\right)^{T}\right]^{T} \\
A(s) & =\left[I_{q+1}, s I_{q+1}, \ldots, s^{n-2} I_{q+1}\right]^{T} \\
\omega_{60}(t) & =\left[\psi_{1}^{T}(t), \psi_{2}^{T}(t), \ldots, \psi_{m}^{T}(t)\right]^{T} \in R^{m(q+1)} .
\end{aligned}
$$

\subsection{Adaptive Control Design}

Based on (2.6) and (4.5), the desired adaptive controller is

$$
\begin{aligned}
v_{0}(t)= & \theta_{1}^{T} \omega_{1}(t)+\theta_{2}^{T} \omega_{2}(t)+\theta_{20} y(t)+\theta_{3} r(t) \\
& +\theta_{6}^{T} \omega_{6}(t)+\theta_{60}^{T} \omega_{60}(t),
\end{aligned}
$$

where $\theta_{1}, \theta_{2}, \theta_{20}, \theta_{3}, \theta_{6}, \theta_{60}$ are estimates of $\theta_{1}^{*}, \theta_{2}^{*}, \theta_{20}^{*}, \theta_{3}^{*}$, $\theta_{6}^{*}, \theta_{60}^{*}$, respectively, and

$$
\omega_{1}(t)=\frac{a(s)}{\Lambda(s)}\left[v_{0}\right](t), \omega_{2}(t)=\frac{a(s)}{\Lambda(s)}[y](t) .
$$

Using (2.11), we can derive

$$
\begin{aligned}
v_{0}(t) & =\theta_{1}^{* T} \frac{a(s)}{\Lambda(s)}\left[v_{0}\right](t)+\theta_{2}^{* T} \frac{a(s)}{\Lambda(s)}[y](t)+\theta_{20}^{*}[y](t) \\
& +\theta_{3}^{*} P_{m}(s)[y](t)-\frac{\left(\Lambda(s)-\theta_{1}^{* T} a(s)\right) P(s)}{\Lambda(s) k_{p} Z_{a}(s)}[\bar{y}](t)
\end{aligned}
$$

From (4.7) and (4.9), we have the tracking error equation as

$$
\theta_{3}^{*} P_{m}(s)\left[y-y_{m}\right](t)=\tilde{\theta}^{T}(t) \omega(t)
$$

where $\tilde{\theta}(t)=\theta(t)-\theta^{*}$,

$$
\begin{aligned}
\theta(t) & =\left[\theta_{1}^{T}(t), \theta_{2}^{T}(t), \theta_{20}(t), \theta_{3}(t), \theta_{6}^{T}(t), \theta_{60}^{T}(t)\right]^{T} \\
\theta^{*} & =\left[\theta_{1}^{* T}, \theta_{2}^{* T}, \theta_{20}^{*}, \theta_{3}^{*}, \theta_{6}^{* T}, \theta_{60}^{* T}\right]^{T} \\
\omega(t) & =\left[\omega_{1}^{T}(t), \omega_{2}^{T}(t), y(t), r(t), \omega_{6}^{T}(t), \omega_{60}^{T}(t)\right]^{T} .(4
\end{aligned}
$$

Based on the error equation (4.10), we introduce

$$
\begin{aligned}
& \zeta(t)=W_{m}(s)[\omega](t) \\
& \xi(t)=\theta^{T}(t) \zeta(t)-W_{m}(s)\left[\theta^{T} \omega\right](t) \\
& \varepsilon(t)=e(t)+\rho(t) \xi(t)
\end{aligned}
$$

where $\rho(t)$ is the estimate of $\rho^{*}=1 / \theta_{3}^{*}$, and choose

$$
\begin{aligned}
& \dot{\theta}(t)=-\frac{\operatorname{sign}\left[\theta_{3}^{*}\right] \Gamma \zeta(t) \varepsilon(t)}{1+\zeta^{T} \zeta+\xi^{2}}, \Gamma=\Gamma^{T}>0 \\
& \dot{\rho}(t)=-\frac{\gamma \xi(t) \varepsilon(t)}{1+\zeta \zeta \zeta+\xi^{2}}, \gamma>0
\end{aligned}
$$

as the adaptive laws for $\theta(t)$ and $\rho(t)$.

This leads to a stable adaptive control system for which the following properties can be proved.

Theorem 4.1 The adaptive controller (4.7) with adaptive laws (4.15) and (4.16), applied to the plant (2.1) with variant actuator failures (2.2), ensures closed-loop signal boundedness and output tracking: $\lim _{t \rightarrow \infty}\left(y(t)-y_{m}(t)\right)=0$.

Remark 4.1 To handle actuator failures with variant and parametrizable failure values described by (2.2), the adaptive version of the term $\theta_{4}^{*}(t)$ in the control law (2.6) is designed from a dynamic compensator, based on a linear parametrization of the unknown parameters appearing bilinearly: one set of parameters from the nominal feedforward compensator $\theta_{1}^{* T} \frac{a(s)}{\Lambda(s)}$ and the other set from the failure signals $\bar{u}_{j}(t)$. It follows from stable plant-model matching that the effect of parameter jumping at the actuator failure time instants decays to zero exponentially and can ignored in the analysis.

Remark 4.2 In the parametrization (4.5), the dimensions of the parameter vectors $\theta_{6}^{*}$ and $\theta_{60}^{*}$ can be reduced.

For $\theta_{60}^{*}$, since the first components of $\psi_{i}(t), i=1,2, \ldots, m$, are all equal to 1 , the corresponding components in $\theta_{60}^{*}$ can be combined as one single parameter, with the first components of $\psi_{i}(t), i=2, \ldots, m$, being deleted from $\omega_{60}(t)$.

For $\theta_{6}^{*}$ and $\omega_{6}(t)$, the first components of $\frac{A(s)}{\Lambda(s)}\left[\psi_{i}\right](t)$, $i=1,2, \ldots, m$, are all the same (which converges to the constant $\frac{1}{\Lambda(0)}$ and can be replaced by $\frac{1}{\Lambda(0)}$ ), and the corresponding components in $\theta_{6}^{*}$ can be combined as one single parameter, with the first components of $\frac{A(s)}{\Lambda(s)}\left[\psi_{i}\right](t), i=2, \ldots, m$, being deleted from $\omega_{6}(t)$. The $(k(q+1)+1)$ th components of $\frac{A(s)}{\Lambda(s)}\left[\psi_{i}\right](t), i=1,2, \ldots, m$, are all the same (equal to 0 ), for $k=1, \ldots, n-2$, which can be deleted from $\omega_{6}(t)$, with the corresponding components in $\theta_{6}^{*}$ being also deleted from $\theta_{6}^{*}$. 
Remark 4.3 If in the expression (2.2), a more compact form is used with $q=q_{j}$ for each $j$, it can be similarly handled with a modification to the parametrization (4.5).

The signals $f_{j l}(t)$ in (2.2), $l=1,2, \ldots, q$, are also allowed to be system dependent, for example, $f_{j l}(t)=f_{j l}(t, y)$ as functions of the output $y$. This includes a class of nonlinear systems which can be handled by the developed approach.

\subsection{Alternative Parametrizations}

The adaptive compensation scheme has been derived based on the actuator failure model (2.2) in which the "basis" functions $f_{j l}(t)$ are assumed to be known so that the term $\theta_{4}^{*}(t)=$ $-\sum_{j=j_{1}, \ldots, j_{p}} \frac{k_{p j} Z_{j}(s) P_{a}(s)}{k_{p} \Lambda(s)}\left[\bar{u}_{j}\right](t)$ in (4.3) can be parametrized as $\theta_{6}^{* T} \omega_{6}(t)+\theta_{60}^{* T} \omega_{60}(t)$ as given in (4.5).

If the basis functions $f_{j l}(t)$ are sinusoidal functions, the term $\theta_{4}^{*}(t)$ can be approximately parametrized as $\theta_{4}^{*}(t)=$ $\theta_{7}^{* T} \omega_{7}(t)$ for some parameter vector $\theta_{7}^{*}$ and regressor vector $\omega_{7}(t)$ which contains all needed sin and cos functions with different frequencies and whose dimension does not depend on the plant order $n$. In this case, the approximation error converges to zero exponentially.

Another case is with $\bar{u}_{j}$ being piecewise constant whose values are unknown but the time instants when $\bar{u}_{j}$ changes values are known. If the number of unknown constant values (which may repeat themselves) is finite, then an exact parametrization of $\theta_{4}^{*}(t)$ can be obtained, similar to that in (4.5). If there is a time delay in the knowledge of the time instants when $\bar{u}_{j}$ changes values, then there is an error in parametrizing $\theta_{4}^{*}(t)$ and this error decays exponentially until the next time instant when a different failure constant occurs which causes a new transient error if a new time delay exists. The magnitude of such errors is proportional to the magnitude of those time delays. If the number of unknown constant values is infinite, we can only parametrize a finite of them, leading to an parametrization error. If we choose $\theta_{4}^{*}$ as a scalar piecewise constant for parametrization, then there is a transient error at each time the failure value changes. For either a finite or infinite number of failure values, this case can be considered as the case with consecutive failures. If the failure value becomes constant after a certain time, then the parametrization error decays to zero exponentially.

When the failure basis functions $f_{j l}(t)$ in (2.2) are unknown, we have to use some known functions to approximate the failure signal $\bar{u}_{j}(t)$. Such known functions should have certain desirable approximation properties. If $\bar{u}_{j}(t)$ is periodic, we can use a linear combination of sinusoidal signals to approximate it (as from the Fourier series theory) so that an approximate parametrization of $\theta_{4}^{*}(t)$ can still be obtained. If $\bar{u}_{j}(t)$ is an arbitrary unknown signal, then a suitable parametrization of $\theta_{4}^{*}(t)$ may not be possible. We should note that a suitable parametrization of $\theta_{4}^{*}(t)$ is the key to effective adaptive failure compensation which, in addition to closedloop stability, improves system tracking performance in the presence of actuator failure and plant parameter uncertainties.
Such an actuator failure compensation design is also applicable to the case when a failed actuator can recover and other actuators become failed, as long as at any given time there is at least one actuator which is not failed.

Finally, we note that redundancy is always needed for failure compensation and the capacity of unfailed actuators for failure compensation is necessary for a desired control performance. The key issue is how to make use of them in the presence of system and failure uncertainties, to which adaptive compensation as developed is a desirable solution.

\section{Simulation Study}

As an illustrative example, we use the lateral dynamics model of a Boeing 747 airplane [6] as the controlled plant to which the adaptive actuator failure compensation control scheme is applied. Unlike that in [8] where only a constant value failure model was considered, in this study, we study adaptive compensation of variant value actuator failures.

Plant model. The linearized lateral dynamics of Boeing 747 with two augmented actuation vectors is described as

$$
\begin{aligned}
\dot{x}(t) & =A x(t)+B u(t), y(t)=x_{2}(t)=y_{r}(t) \\
x(t) & =\left[\beta, y_{r}, p, \phi\right]^{T}, B=\left[b_{1}, b_{2}, b_{3}\right]
\end{aligned}
$$

where $\beta$ is the side-slip angle, $y_{r}$ is the yaw rate, $p$ is the roll rate, $\phi$ is the roll angle, $y$ is the system output, which is the yaw rate in this case, and $u$ is the control input vector, which contains three control signals: $u=\left[u_{1}, u_{2}, u_{3}\right]^{T}$ to represent three rudder servos: $\delta_{r 1}, \delta_{r 2}, \delta_{r 3}$, from a three-piece rudder for compensation of actuator (rudder piece) failures.

From the data in [6], in horizontal flight at $40,000 \mathrm{ft}$ and nominal forward speed $774 \mathrm{ft} / \mathrm{sec}$ (Mach 0.8), the Boeing 747 lateral-perturbation dynamics matrices are

$$
\begin{aligned}
A & =\left[\begin{array}{cccc}
-0.0558 & -0.9968 & 0.0802 & 0.0415 \\
0.598 & -0.115 & -0.0318 & 0 \\
-3.05 & 0.388 & -0.465 & 0 \\
0 & 0.0805 & 1 & 0
\end{array}\right] \\
b_{1} & =\left[\begin{array}{c}
0.00729 \\
-0.475 \\
0.153 \\
0
\end{array}\right], b_{2}=\left[\begin{array}{c}
0.01 \\
-0.5 \\
0.2 \\
0
\end{array}\right], b_{3}=\left[\begin{array}{c}
0.005 \\
-0.3 \\
0.1 \\
0
\end{array}\right]
\end{aligned}
$$

where $b_{2}$ and $b_{3}$ are the augmented actuation vectors for actuator failure compensation. For this plant, it can be verified that Assumption (A.1) is satisfied and that $\operatorname{sign}\left[\theta_{3}^{*}\right]=-1$.

Simulation results. We considered the case where $u_{2}$ failed at $t=30 \mathrm{sec}: u_{2}(t)=u_{2}(30)+0.01 \sin t \mathrm{rad}$, for $t \geq$ $30 \mathrm{sec}$, while $u_{1}=u_{3}=v_{0}$ did not fail.

We used: $\Gamma=5 I, \gamma=5, W_{m}(s)=\frac{1}{7} s+3, \Lambda(s)=(s+$ $1)^{3}, y(0)=0.02, y_{m}(0)=0,\left[\theta_{1}^{T}(0), \theta_{2}^{T}(0), \theta_{20}(0), \theta_{3}(0)\right]=$ $[0.8734,2.7249,2.4537,-1.8387,-5.128,-3.9069,4.1652$, -0.7765 ], and $\rho(0)=-1.2878$ (and zero initial values for 

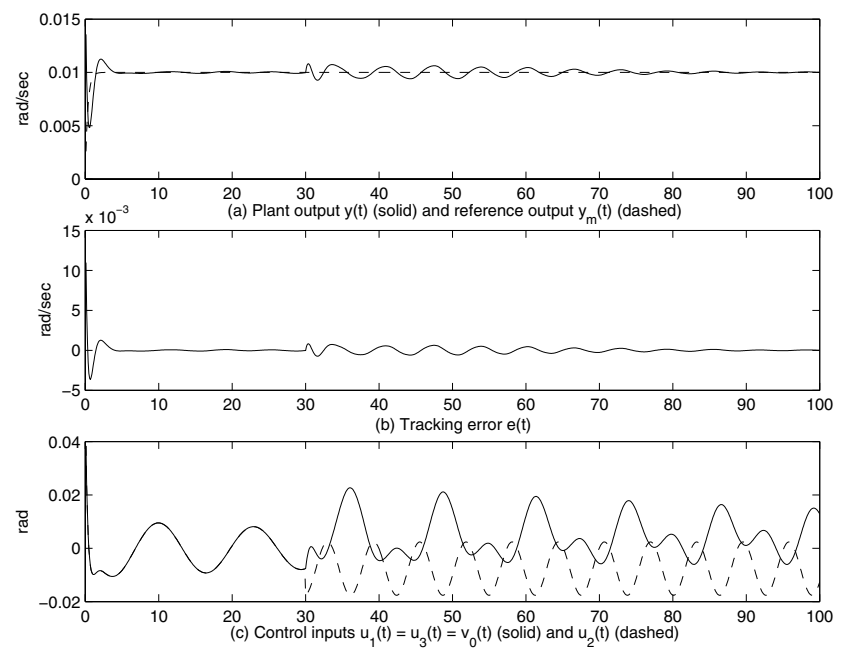

Figure 5.1: Adaptive system response for $r(t)=0.03$.
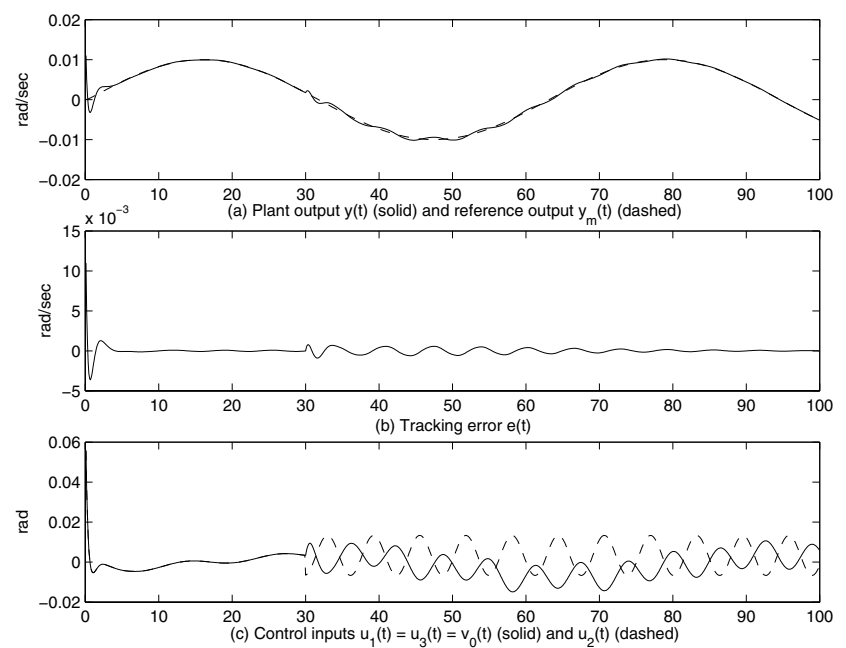

Figure 5.2: Adaptive system response for $r(t)=0.03 \sin 0.1 t$.

$\theta_{6}$ and $\left.\theta_{60}\right)$. The simulation results are shown in Figure 5.1 for $r(t)=0.03$ and in Figure 5.2 for $r(t)=0.03 \sin (0.1 t)$.

These typical results verify the desired system performance: the closed-loop system is stable, and the output tracking error $e(t)=y(t)-y_{m}(t)$, experiencing a transient at the actuator failure time instant, converges to very small value. The adaptive control signals from the unfailed actuators $u_{1}$ and $u_{3}$ are able to compensate the uncertain failure of $u_{2}$ (whose failure information: $j=2, u_{2}(30), t_{2}=30 \mathrm{sec}$ and $d_{2 l}=0.01$, is unknown to the adaptive compensation controller) (the compensation action can be seen from the plots).

\section{Concluding Remarks}

To handle actuator failures with variant and parametrizable failure values, a linear parametrization of the bilinear parameters (one set from the nominal feedforward compensator and the other set from the failure signals) is needed for adaptive failure compensation with desired stability and tracking performance. A relative degree condition is also crucial for output rejection of mismatched input uncertainties caused by such time-varying actuator failures. This result applies to systems with inherent nonlinearities and can also be developed for MIMO and nonlinear system cases.

\section{References}

[1] F. Ahmed-Zaid, P. Ioannou, K. Gousman, and R. Rooney, "Accommodation of failures in the F-16 aircraft using adaptive control," IEEE Control Systems magazine, vol. 11, no. 1, pp. 73-78, 1991.

[2] M. Bodson and J. E. Groszkiewicz, "Multivariable adaptive algorithms for reconfigurable flight control," IEEE Trans. on Control Systems Technology, vol. 5, no. 2, pp. 217-229, 1997.

[3] J. D. Boskovic, S.-H. Yu, and R. K. Mehra, "A stable scheme for automatic control reconfiguration in the presence of actuator failures," Proceedings of the 1998 $A C C$, pp. 2455-2459.

[4] J. D. Boskovic, S.-H. Yu, and R. K. Mehra, "Stable scheme adaptive fault-tolerant control of overactuated aircraft using multiple models, switching and tuning," Proceedings of the 1998 AIAA Guidance, Navigation and Control Conference, vol. 1, pp. 739-749, 1998.

[5] J. Chen and R. Patton, Robust Model-based Fault Diagnosis for Dynamic Systems, Kluwer, 1998.

[6] G. F. Franklin, J. D. Powell, and A. Emani-Naeini, Feedback Control of Dynamic Systems, 3rd ed., Addison Wesley, 1994.

[7] L. H. Mutuel, and J. L. Speyer, "Fault-tolerant estimation", Proceedings of the 2000 ACC, pp. 3718-3722.

[8] G. Tao, S. H. Chen and S. M. Joshi, "An adaptive failure compensation controller using output feedback," IEEE Trans. on Automatic Control, vol. 47, no. 3, pp. 506-511, 2002.

[9] A. T. Vemuri and M. M. Polycarpou, "Robust nonlinear fault diagnosis in input-output systems," International Journal of Control, vol. 68, no. 2, pp. 343-360, 1997.

[10] H. Wang, Z. J. Huang, and S. Daley, "On the use of adaptive updating rules for actuator and sensor fault diagnosis," Automatica, vol. 33, pp. 217-225, 1997.

[11] K. Wise, J. S. Brinker, A. J. Calise, D. F. Enns, and M. R. Elgersma, "Direct adaptive reconfigurable flight control for a tailless advanced fighter aircraft," Int. Journal of Robust and Nonlinear Control, vol. 9, pp. 999-1009, 1999.

[12] X. Zhang, T. Parisini and M. M. Polycarpou, "Adaptive fault-tolerant control of nonlinear uncertain systems: An information-based diagnostic approach," IEEE Trans. on Automatic Control, vol. 49, no. 8, pp. 1259-1274, 2004. 